\title{
Pathology of Metastatic Tumors to Bone: Effects of Decalcification as Experienced at a Single Cancer Center \\ Evita B Henderson-Jackson ${ }^{1}$ and Farah K Khalil2*
}

${ }^{1}$ Department of Pathology and Cell Biology, University of South Florida, Bruce B Downs Blvd, Tampa, Florida, USA

${ }^{2}$ Department of Anatomic Pathology, H. Lee Moffitt Cancer Center \& Research Institute, Magnolia Drive, Tampa, Florida, USA

\begin{abstract}
Background: A frequent consequence of renal cancer is bone metastasis which has devastating effects on patients. Bone metastases are identified radiologically and confirmed histologically by pathologists. In order to evaluate bone tissue microscopically, the tissue has to be decalcified. The decalcification process may damage tissue morphology and routine staining quality.

Methods: In this short report, we will review our institution's collection of bone specimens submitted for decalcification from January 2011 to August 2011 and select biopsy-proven metastatic tumors. Our objective is to briefly describe the types of malignancies commonly associated with metastasis to the skeletal system within our institution and report upon the advantages and disadvantages encountered in our pathology department when processing bone tissue.

Results: Eighty-six bone specimens were identified with metastatic tumors from 83 patients (34 males and 49 females). The most common primary sites were breast, lung, kidney, and prostate. The frequent skeletal site for metastatic spread was the vertebral column.

Conclusions: While the decalcification of bone tissue is labor-intensive and time-consuming, the histologic quality and integrity of immunohistochemical staining of our institution's bone specimens with metastatic tumors are excellent.
\end{abstract}

Keywords: Decalcification; Bone; Metastasis; Kidney; Renal; Cancer

\section{Introduction}

Bone metastases are a frequent complication of renal cancer [1]. Breast and prostate cancer are the most common carcinomas to develop bone metastases, with an incidence of $65-75 \%$ and $68 \%$, respectively [2]. In addition, carcinomas of lung, kidney, and thyroid give rise to bone metastases in approximately $30-40 \%$ of cases [1]. In contrast, gastrointestinal tract carcinomas rarely produce bone metastases $(<10 \%)$ [3]. The primary problems that arise from bone metastases are pain, pathological fractures, spinal cord compression, hypercalcemia, and bone marrow suppression [1].

\section{Decalcification Process}

Bone, a specialized connective tissue, is composed of a collagenous and non-collagenous matrix proteins strengthened by deposits of calcium salts that play an essential role in the hard, rigid architecture and function of bone $[3,4]$. In order to study bone pathology, bone tissue is routinely placed within a formalin fixative followed by a decalcification procedure to remove the calcium salts. If a bone specimen is placed into a decalcification solution without prior formalin fixation the tissue will be macerated destroying tissue morphology and nuclear staining [5]. Removal of the calcium salts makes the bone tissue pliable and flexible which is necessary in order to section the tissue for microscopic evaluation. The decalcification procedure can be a lengthy process that may take several days or even weeks depending on the size of the bone. In our pathology department, once the tissue is submitted for decalcification the specimen is recorded into a log book detailing the date and time the specimen is placed into and removed from the Decal solution. Our pathology department uses a Decal solution named Nitrical which contains deionized water and nitric acid. Specimens in Decal solution are checked daily for pliability and softness. The Decal solution is changed on a regular basis due to the fact that the calcium ions that have been removed may saturate the solution preventing further decalcification. Occasionally, extremely rigid tissue may be processed within a microwave histoprocessor in order to decrease the amount of time for decalcification. Experienced histotechnologists test the completeness of decalcification through bending the tissue. Larger, denser bone specimens may be evaluated with X-ray radiographs to determine if the specimen is appropriately decalcified [5]. When the decalcification process is complete, the tissue is thoroughly washed in running tap water for 15-30 minutes to rinse off residual acid and neutralize the tissue. Appropriately size cut sections $(0.5 \mathrm{~cm}$ in thickness overall size and volume not as critical as thickness) of bone tissue adequately formalin-fixed and decalcified are at this point able to be processed, embedded, and sectioned for light microscopy. All solutions utilized in the decalcification process are changed daily [6].

\section{Results}

From January 2011 to August 2011 approximately 3076 specimens were submitted for decalcification according to the log book of which 86 specimens were identified with bone metastasis consisting of a total of 83 patients. Primary bone lesions (benign and malignant) and hematopoietic disorders arising within bone were excluded. Thirtyfour patients were male (41\%) [age range $34-83$ years old, average age 61 ] and 49 were female (59\%) [age range 33-80 years old, average age 60]. The four most common primary sites were breast (24\%), lung (19\%), renal (13\%), and prostate (6\%) (Table 1).

The majority of the breast carcinomas were of ductal origin (95\%)

*Corresponding author: Farah K Khalil, Department of Anatomic Pathology, $\mathrm{H}$. Lee Moffitt Cancer Center \& Research Institute, 12902 Magnolia Drive, Tampa FL 33612, Florida, Tel: 813-745-3270; E-mail: Farah.khalil@moffitt.org

Received: March 23, 2016; Accepted: April 10, 2016; Published: April 13, 2016

Citation: Henderson-Jackson EB, Khali FK (2016) Pathology of Metastatic Tumors to Bone: Effects of Decalcification as Experienced at a Single Cancer Center. Oncol Cancer Case Rep 1: 112.

Copyright: (C) 2016 Henderson-Jackson EB, et al. This is an open-access article distributed under the terms of the Creative Commons Attribution License, which permits unrestricted use, distribution, and reproduction in any medium, provided the original author and source are credited. 
with the exception of one case of metastatic lobular carcinoma (5\%). The lung carcinomas were primarily adenocarcinoma type (69\%) with a minor portion of squamous cell type (31\%). The spine (49\%) was a frequent site for metastatic spread. Of note, carcinomas of breast, renal and lung were frequently identified in the spine. Other sites of the skeletal system identified with metastases include the femoral head (10\%), femur (9\%), iliac (5\%), tibia (5\%), pelvic (4\%), sternum $(4 \%)$, rib $(4 \%)$, humerus $(2 \%)$, sacrum $(2 \%)$, parietal $(2 \%)$, ischium $(1 \%)$, hip (1\%), frontal (1\%), and acetabulum (1\%). Overall, the histologic sections from the metastatic bone lesions demonstrated adequate tissue morphology and staining quality. The malignant nuclear features were

\begin{tabular}{|l|c|}
\hline Type of Malignancy & Number of Cases (\%) \\
\hline Breast cancer & $20(24 \%)$ \\
\hline Lung cancer & $16(19 \%)$ \\
\hline Renal cancer & $10(13 \%)$ \\
\hline Prostate cancer & $5(6 \%)$ \\
\hline Melanoma & $4(5 \%)$ \\
\hline Soft tissue cancer & $4(5 \%)$ \\
\hline Esophageal cancer & $3(3 \%)$ \\
\hline Neuroendocrine cancer & $3(3 \%)$ \\
\hline Salivary gland cancer & $2(2 \%)$ \\
\hline Bladder cancer & $2(2 \%)$ \\
\hline Skin cancer & $2(2 \%)$ \\
\hline Endometrial cancer & $1(1 \%)$ \\
\hline Liver cancer & $1(1 \%)$ \\
\hline Colorectal cancer & $1(1 \%)$ \\
\hline Thyroid cancer & $1(1 \%)$ \\
\hline Rectal cancer & $1(1 \%)$ \\
\hline Head \& Neck cancer & $1(1 \%)$ \\
\hline Pancreatic cancer & $1(1 \%)$ \\
\hline Cholangiocarcinoma & $1(1 \%)$ \\
\hline Malignancy of unknown origin & $5(6 \%)$ \\
\hline Other & $2(2 \%)$ \\
\hline
\end{tabular}

Table 1: Types of metastatic bone lesions encountered at $\mathrm{H}$ Lee Moffitt cancer center.

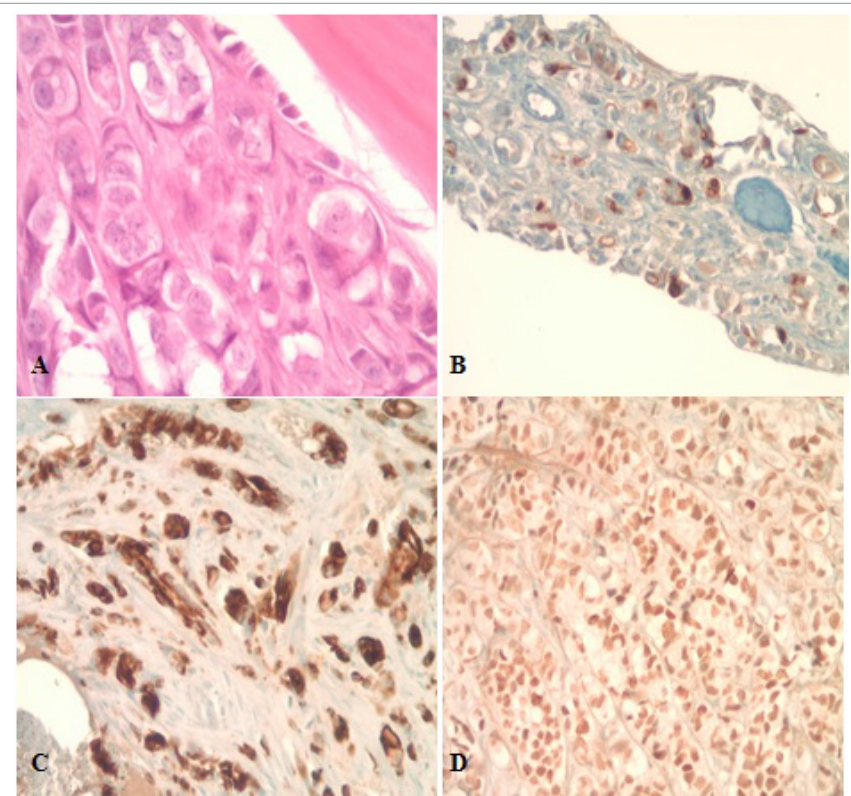

Figure 1: A. Invasive ductal carcinoma of the breast metastatic to bone (high power, 20X); B. Breast 2 immunohistochemical stain is positive; C. Cytokeratin 7 immunostain is positive; $\mathrm{D}$. Estrogen receptor immunostain is positive.

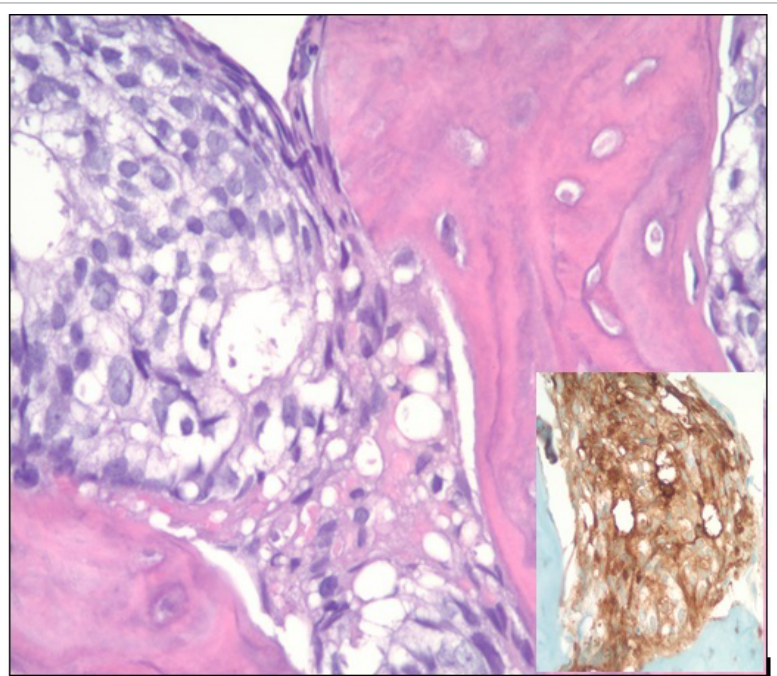

Figure 2: Prostatic adenocarcinoma infiltrating bone (H\&E stain, 20X); Inset: PSA immunohistochemical stain demonstrates positivity in tumor cells.

easily detected. The metastatic breast carcinomas identified infiltrating bony trabeculae were composed of glandular-appearing epithelial cells with moderate to marked nuclear pleomorphism, prominent nucleoli, and numerous mitotic figures (Figure 1). Immunohistochemistry confirmed the diagnosis in most cases and remaining cases had compatible clinical history supporting the diagnoses. The cases of metastatic prostate cancer revealed infiltrating glands or solid nests of malignant cells around bone with enlarged, hyperchromatic nuclei with prominent nucleoli (Figure 2). Immuohistochemical analysis with PSA (Prostate specific antigen) and PSAP (Prostatic acid phosphatase) confirm the diagnosis in $60 \%$ of cases and the remaining $40 \%$ were histologically compatible with prostatic origin.

There were five cases (6\%) of metastatic carcinoma of unknown origin. Furthermore, additional work-up utilizing immunohistochemistry to classify the type of carcinoma in these cases were inconclusive. Two cases comprised of a poorly differentiated carcinoma were immunoreactive for cytokeratin AE1/AE3 while additional immunostains helpful in determining the origin of certain types of malignancies were non-specific and radiologic findings revealed multiple lesions throughout the body in one case and no identifiable lesions in the other case. The third case illustrated a poorly differentiated carcinoma with histologic features of adenocarcinoma, however, clinically the patient had numerous lesions throughout the body and a single source could not be determined. In addition, the metastatic tumor was immunoreactive for pancytokeratin AE1/AE3, cytokeratin 7, and MOC-31 while other more tumor-origin specific immunostains were negative. Basically, immunostains were only able to confirm the presence of carcinoma without determining origin. The last two cases were biopsies from separate areas in the lumbar spine from a single patient that histologically appeared to be a metastatic carcinoma with squamoid features suggestive of urothelial carcinoma (Figure 3). However, the tumors were only pancytokeratin AE1/AE3 positive and negative for immunostains consistent with urothelial origin (cytokeratin 7 and 20 negative). Clinically the patient had a history of prostate cancer and the patient's genitourinary system clinically appeared normal without definite masses or lesions. The sources of these tumors were not able to be definitively identified.

There were two cases (2\%) classified as "other" in which immunohistochemical analysis was non-specific in identifying the 


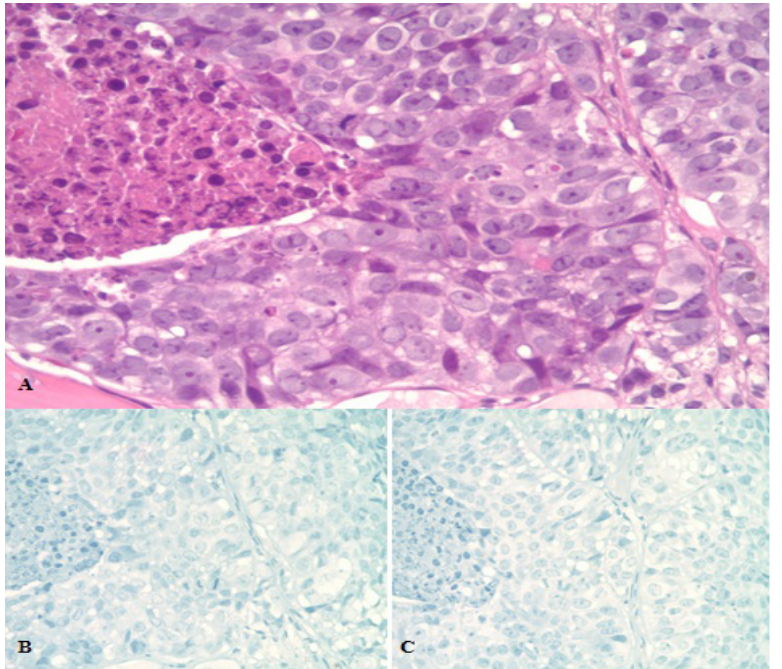

Figure 3: A. Metastatic carcinoma with squamoid features suspected to be urothelial carcinoma in a patient without a bladder lesion and only a history of prostate cancer. B. Cytokeratin 7 is negative. C. PSA is negative.

origin of the metastatic tumor, however, clinical history along with radiographic imaging aided with a possible source for the malignancy. One case revealed a poorly differentiated carcinoma with necrosis only reactive for cytokeratin 7 while the other case histologically and immunohistochemically demonstrated an adenosquamous carcinoma. Both tumors were negative for TTF-1. However, both patients had a suspicious lung mass. Unfortunately, only one patient had a lung biopsy and the tumor from the lung was focally TTF-1 positive in support of a malignancy of lung origin. However, the bone metastasis was negative for TTF-1. The histology of the lung tumor was similar to the bone metastasis clinically supporting a metastatic lung carcinoma.

\section{Discussion}

Tissue morphology upon the hematoxylin \& eosin (H\&E) stained slides of the specimens displayed intact architecture and adequate cellular preservation. All of our specimens revealed excellent discrimination between normal bone tissue and infiltrating malignant cells. Immunohistochemical studies performed on the decalcified tissue were able to further classify the type of malignancy when possible. The immunostaining quality of a majority of the specimens was excellent and only a few cases showed less than optimal staining quality. The few cases of unknown origin showed immunoreactivity with one or two non-specific immunostains while the additional more specific antibody stains showed no immunoreactivity. This may be due to the fact that the cases comprised of poorly differentiated malignant neoplasms which may lose certain antigenic markers but effects from decalcifying agents cannot be excluded. Decalcifying agents including strong acids, weak acids, or chelating agents may adversely affect cytological detail and routine staining quality, particularly when tissue is placed within a decalcification solution for a long period of time [7]. Acid decalcifying agents dissolve calcium salts in an acid solution such as hydrochloric acid, nitric acid, formic acid, or acetic acid [5]. Some decalcifying solutions contain formalin which may increase the possibility of aldehyde groups in the tissue and block immunoreactions [5]. EDTA (Ethylenediaminetetraacetic Acid), a common chelating agent used in decalcification, combines with calcium ions to form soluble, nonionized compounds. It can decalcify a $5 \mathrm{~mm}$ thick portion of bone within 24-48 hours [5]. Our pathology department uses a nitric acid decalcification solution that appears to perform adequately on our bone specimens rarely causing problems with tissue histology and immunoreactivity.

Our findings are unique, in that, there are rare publications detailing the processing, histology, and use of immunohistochemistry in bone specimens within a tertiary care center. Immunohistochemistry confirmed the diagnosis in most cases (81\%).

We show that the immunohistochemical reactivity was preserved in most of our decalcified specimens. Although, Matthews et al reported that tissue treated with decalcifying agents may exhibit variable immunoreactivity and impaired counterstaining especially with the use of strong mineralic acids [8]. Overall, laboratories must figure out which type of decalcifying agent to use with the type of bone specimens received, accounting for the amount of mineralized tissue present, which would best ensure an accurate diagnosis. Our facility decalcifies bone marrow biopsies for only one hour whereas bone biopsies and bone resection specimens decalcify until deemed appropriate for processing which could be a few hours, a couple of days or even a few weeks depending upon the size and type of bone, either cortical (dense compact bone) or cancellous (spongy bone), being decalcified. Set standards for decalcification of bone do not exist at this time. Our institution's unique method in processing bone specimens for optimal histology and use for immunohistochemistry may be a start, particularly, when oncologic diagnoses are within the differential.

Bone metastases are common in patients with advanced cancers and have devastating effects. In our review of metastatic bone lesions at our institution, carcinoma of the breast frequently metastasizes to the bone which is consistent with prior reports. Prostate cancer commonly metastasizes to the bone but in our patient population, there were only a handful of cases of metastatic prostate cancer. This may be reflective of the patient population seen at $\mathrm{H}$ Lee Moffitt Cancer Center. Other carcinomas recognized to demonstrate bone metastases like carcinoma of the lung, kidney, and thyroid were present in our review. Furthermore, several tumors rarely identified infiltrating bone were seen at our institution which include pancreatic cancer, cholangiocarcinoma, esophageal cancer, endometrial cancer, and even salivary gland cancer. Overall, our institution's decalcified specimens with metastatic malignancy are not difficult to identify. Our decalcification procedure appears to be efficient and adequate for our pathologists to make accurate diagnoses of malignancy. We are able to predict the primary site in most cases and suggest possible primary sites for the remaining cases where histology is not definitive of origin and immunostains are not specific. Clinical history and radiologic imaging reports are extremely helpful in such cases. The abilities of our pathology department to utilize special solutions and processing equipment to study bone tissue aid in the care and treatment of our patients.

\section{Conclusion}

Although the processing of bone specimens is complex, laborintensive, and time-consuming, our institution is able to provide pathologists with optimally processed bone specimens exhibiting excellent histology. In addition, the integrity of immunohistochemical staining of our bone specimens with metastases is preserved. Our next steps include acquiring additional extended data to evaluate the size of bone tissue, type of bone, and density of bone in our specimens with metastases to determine the possibility of defining a standard to guide laboratories in appropriately processing bone specimens from different locations and bone specimens of different size, especially for oncologic 
Citation: Henderson-Jackson EB, Khalil FK (2016) Pathology of Metastatic Tumors to Bone: Effects of Decalcification as Experienced at a Single Cancer Center. Oncol Cancer Case Rep 1: 112.

cases. Further studies looking into the effect of different metastatic tumor subtypes and the surrounding bone environment are warranted as well.

\section{References}

1. Rubens R (1998) Bone metastases--the clinical problem. European Journal of Cancer 34: 210-213.

2. Perez J, Machiavelli M, Leone BA, Romero A, Rabinovich MG, et al. (1990) Bone-only versus visceral-only metastastic pattern in breast cancer: analysis of 150 patients. Am J Clin Oncol 13: 294-298.

3. Buijs J, Van der Pluijm G (2009) Osteotropic cancers: from primary tumor to bone. Cancer Letters 273: 177-193.
4. Wittenburg G, Volkel C, Mai R, Lauer G (2009)Immunohistochemical comparison of differentiation markers on paraffin and plastic embedded human bone samples. Journal of Physiology and Pharmacology 60: 43-49.

5. An YH, Martine KL (2003) Handbook of histology methods for bone and cartilage. Totowa, NJ: Humana Press Inc.

6. Sheehan DC, Hrapchak BB (1980) Theory and Practice of Histotechnology (2nd edn)

7. Athanasou NA, Quinn J, Heryet A, Woods CG, McGee O'J (1987) Effect of decalcification agents on immunoreactivity of cellular antigens. J Clin Patho 40: 874-878.

8. Matthews J, Masson G (1984) Influence of decalcifying agents on immunoreactivity of formalin-fixed, paraffin-embedded tissue. Histochem $\mathrm{J} 16$ : 771-787. 